\title{
On manifolds of small degree
}

\author{
Paltin Ionescu
}

\begin{abstract}
Let $X \subset \mathbb{P}^{n}$ be a complex connected projective, non-degenerate, linearly normal manifold of degree $d \leqslant n$. The main result of this paper is a classification of such manifolds. As a by-product of the classification it follows that these manifolds are either rational or Fano. In particular, they are simply connected (hence regular) and of negative Kodaira dimension. Moreover, easy examples show that $d \leqslant n$ is the best possible bound for such properties to hold true. The proof of our theorem makes essential use of the adjunction mapping and, in particular, the main result of [15] plays a crucial role in the argument.
\end{abstract}

Mathematics Subject Classification (2000). Primary 14N25, 14N30; Secondary 14M99, $14 \mathrm{~J} 45$.

Keywords. Embedded projective manifold, small degree, rational manifold, Fano manifold, adjunction mapping.

\section{Introduction}

Let $X \subset \mathbb{P}^{n}$ be a complex connected projective manifold of dimension $r$ and degree $d$. Assume moreover that $X$ is non-degenerate and $d \leqslant n$. The results contained in this paper have the following topological consequence:

$$
\text { If } X \text { is as above, } X \text { is simply connected. }
$$

The bound $d \leqslant n$ is optimal for the validity of $(*)$. Indeed, there exist $r$-dimensional elliptic scrolls in $\mathbb{P}^{2 r}$, of degree $2 r+1$ (see [14], 5.2); they have $b_{1}=2$.

To the best of our knowledge, $(*)$ was not even conjectured before. However, F. L. Zak (unpublished) asked if such manifolds are regular (i.e. if $b_{1}=0$ ).

We would like to mention two related topological ancestors of $(*)$. The first one is (a special case of) the Barth-Larsen theorem (see [3] and, for a singular version, [8])

$$
\text { If } 2 r \geqslant n+1 \text {, then } \pi_{1}(X)=(0) .
$$

The second result is the Fulton-Gaffney-Lazarsfeld theorem about branched coverings of $\mathbb{P}^{r}$ (see [9], [8]):

$$
\text { If } X \rightarrow \mathbb{P}^{r} \text { is a normal finite covering of degree } d \leqslant r \text {, then } \pi_{1}(X)=(0) \text {. }
$$


Note that, for $d \leqslant r,(*)$ follows either from (BL) or from (FGL). We refer to [8] for a very nice discussion of such topological aspects.

To the best of our knowledge, no topological proof of $(*)$ is known. We are able to deduce it from the following geometric result:

If $X$ is as above, then either:

(1) $b_{2}=1$ and $X$ is a Fano manifold, or

(2) $b_{2} \geqslant 2$ and $X$ is rational.

It is well-known that both rational and Fano manifolds are simplyconnected; see [17] for a far-reaching common generalization. So $(*)$ follows from $(* *)$. The first case in (**) may be seen as generic, as it includes all complete intersections of dimension at least three. Assuming Hartshorne's Conjecture for Fano manifolds, we may describe all cases in (1) which are not complete intersections (Corollary 11). Moreover, we shall prove:

Manifolds with $d \leqslant n$ and $b_{2} \geqslant 2$ may be classified completely.

There are 6 infinite series (having arbitrarily large dimension and $\quad(* * *)$ degree) and 14 "sporadic" examples. All turn out to be rational.

The precise list is given in the statement of the main result, see the next section.

The proof of the main theorem will occupy Section 4. It relies on a very detailed study of the adjunction mapping (see e.g. [4], Chapters 9-11 for a complete treatment). Moreover, the main result of [15] plays a key role in the proof. We note that, besides classical adjunction theory, some nontrivial facts coming from Mori theory are also used in [15]. Finally, the classification of manifolds of small $\Delta$-genus (cf. [6], [7], [13]) is also needed.

The present work is a slightly improved version of a paper with the same title that was circulated as Preprint no. 17, IMAR, Bucharest, December 2000.

\section{Statement of the main result}

Our main result is the following:

Theorem. Let $X \subset \mathbb{P}^{n}$ be a connected projective manifold over $\mathbb{C}$, of dimension $r$ and degree $d$. Assume moreover that $X$ is non-degenerate and linearly normal. If $d \leqslant n$, then one of the following holds:

(i) $X$ is Fano, $b_{2}(X)=1$;

(ii) $X$ is Fano and either: 
(a) $2 \leqslant r \leqslant 4,3 \leqslant d \leqslant 8, X$ is a classical del Pezzo manifold with $b_{2}(X) \geqslant 2$ (cf. Theorem B below);

(b) $r=3, d=9, X$ is the Segre embedding of $\mathbb{P}^{1} \times \mathbb{F}_{1}$, where $\mathbb{F}_{1}$ is the blowing-up of $\mathbb{P}^{2}$ in a point, embedded in $\mathbb{P}^{4}$ as a rational scroll of degree 3 ;

(c) $X$ is one of the following scrolls over $\mathbb{P}^{2}$ :

(1) $r=4, d=10, X \simeq \mathbb{P}\left(T_{\mathbb{P}^{2}} \oplus \mathcal{O}_{\mathbb{P}^{2}}(1)\right)$;

(2) $r=4, d=11, X \simeq \mathbb{P}\left(\mathcal{O}_{\mathbb{P}^{2}}(1) \oplus \mathcal{O}_{\mathbb{P}^{2}}(1) \oplus \mathcal{O}_{\mathbb{P}^{2}}(2)\right)$;

(3) $r=5, d=10, X$ is the Segre embedding of $\mathbb{P}^{2} \times \mathbb{P}^{3}$;

(iii) $r \geqslant 2, d \geqslant r, X$ is a scroll over $\mathbb{P}^{1}$ (i.e. a linear section of the Segre embedding of $\left.\mathbb{P}^{1} \times \mathbb{P}^{m}\right)$;

(iv) $r \geqslant 3$ and there is a vector bundle $\mathbb{E}$ over $\mathbb{P}^{1}$, of rank $r+1$ and of splitting type $e=\left(e_{0}, \ldots, e_{r}\right)$, such that, if $L$ denotes the tautological divisor on $\mathbb{P}(\mathcal{E})$ and $F$ denotes a fibre of the projection $\mathbb{P}(\mathscr{E}) \rightarrow \mathbb{P}^{1}, X$ embeds in $\mathbb{P}(\mathcal{E}),\left.L\right|_{X}$ is the hyperplane section divisor and either:

(a) $n=d=2 r-1, e=(1, \ldots, 1,0,0), X \in|2 L+F|$;

(b) $n=d=2 r, e=(1, \ldots, 1,0), X \in|2 L|$;

(c) $n=d=2 r+1, e=(1, \ldots, 1), X \in|2 L-F|$;

(d) $r \geqslant 4, n=2 r+1, d=2 r, e=(1, \ldots, 1), X \in|2 L-2 F|$; equivalently, $X$ is the product of a line and a quadric of dimension $r-1$, in its Segre embedding;

(e) $n=d=2 r+2, e=(1, \ldots, 1,2), X \in|2 L-2 F|$.

Remarks. 1. Except for case (i), all manifolds appearing in the theorem are rational. 2. All cases listed actually occur.

3. Manifolds from case (iv) (b) up to (iv) (e) in the theorem are also Fano.

\section{Conventions and prerequisites}

We follow the customary notation in algebraic geometry (see e.g. [12]). We denote by $X \subset \mathbb{P}_{\mathbb{C}}^{n}$ a complex projective connected manifold. We let $d$ be its degree and $r$ its dimension; $s=n-r$ is the codimension of $X$ in $\mathbb{P}^{n}$. The irregularity of $X$ is by definition $q:=h^{1}\left(X, \mathcal{O}_{X}\right)$ and $H$ will denote a hyperplane section of $X \subset \mathbb{P}^{n}$. We write $\langle Y\rangle$ for the linear span of $Y \subset \mathbb{P}^{n}$. The sectional genus of $X$, denoted $g$, is the genus of the curve $X \cap H_{1} \cap \cdots \cap H_{r-1}$, where $H_{1}, \ldots, H_{r-1}$ are generic hyperplanes in $\mathbb{P}^{n}$. The adjunction formula reads:

$$
2 g-2=(K+(r-1) H) \cdot H^{r-1},
$$


where $K$ is a canonical divisor for $X$.

The $\Delta$-genus of $X$ is by definition

$$
\Delta=d+r-h^{0}\left(X, \mathcal{O}_{X}(H)\right)
$$

and is a non-negative integer.

$X$ is said to be a scroll over the manifold $Y$ if $X \simeq \mathbb{P}(\mathscr{E})$ for some vector bundle $\&$ on $Y$, such that $\mathcal{O}_{X}(H)$ is the tautological line bundle of $\mathbb{P}(\mathscr{E})$. We use Grothendieck's notation for $\mathbb{P}(\mathscr{E})$.

$X$ is said to be a quadric fibration over the smooth curve $C$ if there is a morphism $\pi: X \rightarrow C$ such that the fibres of $\pi$ are quadrics with respect to the embedding induced by $\mathcal{O}_{X}(H)$. It turns out that singular fibres of $\pi$ are ordinary cones (see [13]). In the sequel, we denote by $Q^{r}$ a quadric of dimension $r$.

The adjunction mapping of $X$, denoted below by $\varphi$, is the rational map on $X$ associated with the linear system $|K+(r-1) H|$. See e.g. [4], Chapters 9-11 for a complete study of its properties.

We recall two results on the classification of manifolds of small $\Delta$-genus. The first one is classical (see e.g. [13], Proposition 2.3).

Theorem A. The following are equivalent:

(i) $\Delta=0$;

(ii) $g=0$;

(iii) $X$ is either $\mathbb{P}^{r}, H \in\left|\mathcal{O}_{\mathbb{P}^{r}}(1)\right|$, or a quadric $Q^{r} \subset \mathbb{P}^{r+1}$, or $\mathbb{P}^{2}, H \in\left|\mathcal{O}_{\mathbb{P}^{2}}(2)\right|$, or a scroll over $\mathbb{P}^{1}$.

The next result is due to del Pezzo if $r=2$, to Fano and Iskovskikh for $r=3$ and to Fujita in general (see also [13], Proposition 2.4 for some other characterizations).

Theorem B (Fujita, [6], [7]). Assume that $r \geqslant 2$. The following are equivalent:

(i) $\Delta=1$;

(ii) $X$ is either a classical del Pezzo surface (anticanonical embedding of either $\mathbb{P}^{1} \times \mathbb{P}^{1}$ or $\mathbb{P}^{2}$ blown-up at at most six points) or , if $r \geqslant 3$, one of the following:

(a) a cubic hypersurface;

(b) a complete intersection of type $(2,2)$;

(c) a linear section of the Plücker embedding of the Grassmannian of lines in $\mathbb{P}^{4}$;

(d) the Segre embedding of $\mathbb{P}^{2} \times \mathbb{P}^{2}$;

(e) a hyperplane section of the manifold in (d) (this is $\mathbb{P}\left(T_{\mathbb{P}^{2}}\right)$ );

(f) the Segre embedding of $\mathbb{P}^{1} \times \mathbb{P}^{1} \times \mathbb{P}^{1}$; 
(g) the scroll over $\mathbb{P}^{2}, \mathbb{P}\left(\mathcal{O}_{\mathbb{P}^{2}}(1) \oplus \mathcal{O}_{\mathbb{P}^{2}}(2)\right)$ (this is $\mathbb{P}^{3}$ blown-up at a point);

(h) the Veronese embedding $v_{2}\left(\mathbb{P}^{3}\right)$.

Recall that $X$ is a Fano manifold if $-K$ is ample. We see that the examples listed in Theorem B (which were called classical del Pezzo manifolds in [13]) are all Fano manifolds.

\section{Proof of the theorem}

We begin with the following simple fact.

Lemma 1. Let $C$ be a smooth projective curve of positive genus and let $\mathscr{L} \in \operatorname{Pic}(C)$ with $\operatorname{deg}(\mathcal{L})>0$. Then we have $h^{0}(\mathcal{L}) \leqslant \operatorname{deg}(\mathcal{L})$.

Proof. If $\mathcal{L}$ is special, we may apply Clifford's theorem. If $\mathcal{L}$ is non-special, the result follows from the Riemann-Roch theorem.

Proposition 2. Let $C$ be a smooth projective curve of positive genus and let $\&$ be an ample and spanned vector bundle on $C$. Then we have $h^{0}(\mathscr{E}) \leqslant \operatorname{deg}(\mathcal{E})$.

Proof. We proceed by induction on $e:=\operatorname{rank}(\mathcal{E})$. When $e=1$, we may apply Lemma 1. Assume now $e \geqslant 2$. As $\mathscr{E}$ is ample and spanned, it follows that $h^{0}(\mathscr{E})>e$. So, for $p \in C$, we may find a non-zero section $t \in H^{0}(C, \mathcal{E}(-p))$ which induces an exact sequence:

$$
0 \longrightarrow \mathcal{L} \longrightarrow \mathcal{E} \longrightarrow \mathcal{E}^{\prime} \longrightarrow 0,
$$

where $\mathcal{L} \in \operatorname{Pic}(C), \operatorname{deg}(\mathcal{L}):=l>0$, and $\mathcal{E}^{\prime}$ is ample, spanned and of rank $e-1$. Indeed, $\mathcal{L}^{\vee}$ is the image of $t^{\vee}: \mathcal{E}^{\vee} \rightarrow \mathcal{O}_{C}(-p)$; as $C$ is a smooth curve, this (nontrivial) sheaf of ideals is invertible. We have

$$
\operatorname{deg}(\mathscr{E})-l=\operatorname{deg}\left(\mathcal{E}^{\prime}\right) \geqslant h^{0}\left(\mathcal{E}^{\prime}\right) \geqslant h^{0}(\mathscr{E})-h^{0}(\mathcal{L})
$$

by the induction hypothesis and the cohomology sequence of the above exact sequence. Applying once again Lemma 1 we get $\operatorname{deg}(\mathscr{E}) \geqslant h^{0}(\mathcal{E})$.

Corollary 3. Let $X \subset \mathbb{P}^{n}$ be a scroll over a smooth curve $C$. Assume that $X$ is non-degenerate of degree $d \leqslant n$. Then $C \simeq \mathbb{P}^{1}$.

Proof. Let $X \simeq \mathbb{P}(\mathscr{E})$. If $g(C)>0$, by Proposition 2 we get

$$
n+1 \leqslant h^{0}\left(X, \mathcal{O}_{X}(H)\right)=h^{0}(C, \mathcal{E}) \leqslant \operatorname{deg}(\mathcal{E})=d,
$$

a contradiction. 
Lemma 4. Let $X \subset \mathbb{P}^{n}$ be smooth connected non-degenerate of degree $d$ and dimension $r$, with $d \leqslant n$. Assume moreover that $2 r \leqslant n+1$. Then we have:

(i) $g \leqslant r-1$; and

(ii) $d \geqslant 2 g+1$.

Proof. (i) Let $s:=n-r$ and let $C \subset \mathbb{P}^{s+1}$ be a curve section of $X$. If $H_{C}$ is special, by Clifford's theorem we get

$$
s+2 \leqslant h^{0}\left(C, \mathcal{O}_{C}\left(H_{C}\right)\right) \leqslant \frac{d}{2}+1 \leqslant \frac{r+s}{2}+1,
$$

giving $r \geqslant s+2$. This is a contradiction. So $H_{C}$ is non-special and by Riemann-Roch we get

$$
s+2 \leqslant h^{0}\left(C, \mathcal{O}_{C}\left(H_{C}\right)\right)=d+1-g \leqslant r+s+1-g,
$$

hence $g \leqslant r-1$.

(ii) We get by Riemann-Roch and (i):

$$
d \geqslant s+1+g \geqslant r+g \geqslant 2 g+1 .
$$

Proposition 5. Let $X \subset \mathbb{P}^{n}$ be smooth connected non-degenerate and linearly normal with $d \leqslant n$. Assume that the adjunction mapping $\varphi=\varphi_{|K+(r-1) H|}$ makes $X$ into a scroll over a smooth surface $S$. Then $S \simeq \mathbb{P}^{2}$ and $X$ is one of the following:

(1) $r=4, d=10, X \simeq \mathbb{P}\left(T_{\mathbb{P}^{2}} \oplus \mathcal{O}_{\mathbb{P}^{2}}(1)\right)$;

(2) $r=4, d=11, X \simeq \mathbb{P}\left(\mathcal{O}_{\mathbb{P}^{2}}(1) \oplus \mathcal{O}_{\mathbb{P}^{2}}(1) \oplus \mathcal{O}_{\mathbb{P}^{2}}(2)\right)$;

(3) $r=5, d=10, X \simeq \mathbb{P}\left(\mathcal{O}_{\mathbb{P}^{2}}^{\oplus 4}(1)\right)$, i.e. $X$ is the Segre embedding of $\mathbb{P}^{2} \times \mathbb{P}^{3}$.

Proof. Let $S^{\prime}$ be the smooth surface $X \cap H_{1} \cap \cdots \cap H_{r-2}$, where $H_{i}$ are generic hyperplanes in $\mathbb{P}^{n}$. We first remark that the geometric genus of $S^{\prime}$ is zero. This follows from Lemma 4 (ii) and the adjunction formula for $H_{S^{\prime}}$. The standard exact sequences

$$
\begin{gathered}
0 \longrightarrow \mathcal{O}_{X}(K+(r-2) H) \longrightarrow \mathcal{O}_{X}(K+(r-1) H) \\
\longrightarrow \mathcal{O}_{H}\left(K_{H}+(r-2) H_{H}\right) \longrightarrow 0
\end{gathered}
$$

together with Lemma 1.1 from [13] show that $h^{0}\left(X, \mathcal{O}_{X}(K+(r-1) H)\right)=g-q$ in our case. So, we have $\varphi: X \rightarrow S \subset \mathbb{P}^{g-q-1}$. Let $H_{S}$ be a generic hyperplane section of $S \subset \mathbb{P}^{g-q-1}$ and let $Y:=\varphi^{-1}\left(H_{S}\right)$. Note that $Y$ is a scroll of dimension $r-1$ over the curve $H_{S}$; if we let $d_{Y}$ be its degree, we get $d_{Y}=(K+(r-1) H) \cdot H^{r-1}=2 g-2$ by the adjunction formula. Let $m$ be the dimension of the projective space spanned by $Y$ inside $\mathbb{P}^{n}$. By Barth's theorem (see [2]), the Picard group of $Y$ is cyclic whenever 
$m<2(r-1)-1$. As $Y$ is a scroll, we must have $m \geqslant 2(r-1)-1$. We get, using Lemma 4 (i), that

$$
m \geqslant 2 r-3 \geqslant 2(r-2) \geqslant 2(g-1)=d_{Y} .
$$

So, by Corollary 3 , it follows that $H_{S} \simeq \mathbb{P}^{1}$. The two-dimensional case of Theorem A shows that $q=0$ and one of the following holds:

1. $S=\mathbb{P}^{2}, g=\Delta=3$;

2. $S$ is a scroll over $\mathbb{P}^{1}$;

3. $S$ is the Veronese embedding $v_{2}\left(\mathbb{P}^{2}\right), g=6$.

Recalling the definition $\Delta=d+r-h^{0}\left(X, \mathcal{O}_{X}(H)\right)$, we get

$$
n+r \geqslant d+r \geqslant n+1+\Delta,
$$

giving $r \geqslant \Delta+1$. Now, if we are in case 1, by Proposition 4.7 from [13], it follows that we have the following possibilities for $X$ :

$$
\begin{aligned}
& r=4, d=9,10 \text { or } 11 ; \\
& r=5, d=10, X \text { is the Segre embedding of } \mathbb{P}^{2} \times \mathbb{P}^{3} .
\end{aligned}
$$

Assume that $r=4$, so $X \simeq \mathbb{P}(\mathscr{E})$ for some very ample vector bundle of rank three over $\mathbb{P}^{2}$. If $\ell$ is a line in $\mathbb{P}^{2}$, it follows that $\varepsilon_{\ell}$ has degree 4 and is very ample. So, $\left.\mathcal{E}\right|_{\ell} \simeq \mathcal{O}_{\ell}(1) \oplus \mathcal{O}_{\ell}(1) \oplus \mathcal{O}_{\ell}(2)$, i.e. $\mathcal{E}$ is uniform. One may use the classification from [5]; we find that the case $d=9$ is not possible, while for $d=10$ we get $\mathcal{E} \simeq T_{\mathbb{P}^{2}} \oplus \mathcal{O}_{\mathbb{P}^{2}}$ (1) (equivalently $X$ is a hyperplane section of the Segre embedding of $\mathbb{P}^{2} \times \mathbb{P}^{3}$ ) and for $d=11$ we get $\mathcal{E} \simeq \mathcal{O}_{\mathbb{P}^{2}}(1) \oplus \mathcal{O}_{\mathbb{P}^{2}}(1) \oplus \mathcal{O}_{\mathbb{P}^{2}}$ (2) (this is the blow-up of $\mathbb{P}^{4}$ with center a line).

To finish the proof we only have to show that cases 2 and 3 cannot occur. We use the notation from [12], Chapter V, Section 2. If we are in case 2, we have $S \simeq \mathbb{F}_{e}$, $H_{S}=C_{0}+b F$ with $b>e \geqslant 0$.

We look at the $(r-1)$-dimensional rational scrolls $Y_{0}=\varphi^{-1}\left(C_{0}\right)$ and $Y_{1}=$ $\varphi^{-1}(F)$. Put $d_{i}=\operatorname{deg}\left(Y_{i}\right)$ for $i=0,1$; we get $d_{i} \geqslant r-1$. By Barth's theorem ([2]), if $m_{i}=\operatorname{dim}\left\langle Y_{i}\right\rangle$, we get as above $m_{i} \geqslant 2(r-1)-1$; moreover, since $\Delta\left(Y_{i}, \mathcal{O}_{Y_{i}}(H)\right)=0$ (see Theorem A) we deduce that

$$
d_{i}+r-1=h^{0}\left(Y_{i}, \mathcal{O}_{Y_{i}}(H)\right) \geqslant m_{i}+1 \geqslant 2(r-1),
$$

i.e. $d_{i} \geqslant r-1$. So, we find

$$
2 g-2=\operatorname{deg}(Y)=d_{0}+b d_{1} \geqslant d_{0}+d_{1} \geqslant 2(r-1),
$$

contradicting part (i) of Lemma 4. Case 3 is ruled out by a similar argument.

Next we need a general lemma concerning the geometry of quadric fibrations (see also [14], 6.2). 
Lemma 6. Assume that the adjunction mapping $\varphi: X \rightarrow C \subset \mathbb{P}^{m}$ makes $X$ into a quadric fibration over a smooth curve $C$. Then $m=g-q-1$ and $q$ coincides with the genus of $C$. Moreover, $\mathbb{E}:=\varphi_{*} \mathcal{O}_{X}(H)$ is a spanned vector bundle of rank $r+1$ over $C$. Denote by $\pi: \mathbb{P}(\mathscr{E}) \rightarrow C$ the projection and by $L$ the tautological divisor on $\mathbb{P}(\mathcal{E})$. Then $X$ is embedded in $\mathbb{P}(\mathcal{E})$ in such a way that $\left.L\right|_{X}=H$ and $X \in\left|2 L+\pi^{*} B\right|$ for some divisor $B$ on $C$. Finally, if $a:=\operatorname{deg}(\&)$ and $b:=\operatorname{deg}(B)$, the following formulae hold:

$$
a=1-g+2(q-1)+d \quad \text { and } \quad b=2(g-1)-4(q-1)-d .
$$

Proof. From Lemma 1.1 in [13] and the standard exact sequences

$$
\begin{gathered}
0 \longrightarrow \mathcal{O}_{X}(K+(r-2) H) \longrightarrow \mathcal{O}_{X}(K+(r-1) H) \\
\longrightarrow \mathcal{O}_{H}\left(K_{H}+(r-2) H_{H}\right) \longrightarrow 0
\end{gathered}
$$

it follows that $h^{0}\left(X, \mathcal{O}_{X}(K+(r-1) H)\right)=g-q$. Let $S \subset X$ be a surface section of $X$, i.e. $S=X \cap H_{1} \cap \cdots \cap H_{r-2}$, where $H_{i}$ are generic hyperplanes in $\mathbb{P}^{n}$. By Lefschetz's theorem on hyperplane sections, $X$ and $S$ have the same irregularity. As $S$ is a conic fibration over $C$, it is birationally ruled, so we have $q=q(S)=g(C)$. For any $c \in C$, let $X_{c}$ denote the fibre of $\varphi$ over $c$. Note that $X_{c}$ is a quadric of dimension $r-1$, hence it is linearly normal in its linear span $\mathbb{P}^{r}$. In particular, for any $c \in C$, we have $h^{0}\left(X_{c}, \mathcal{O}_{X_{c}}(H)\right)=r+1$ and $H^{1}\left(X_{c}, \mathcal{O}_{X_{c}}(H)\right)=0$. So $\&$ is a vector bundle by Grauert's theorem; let $\mathcal{E}_{c}$ be its fibre at the point $c$. The canonical diagram

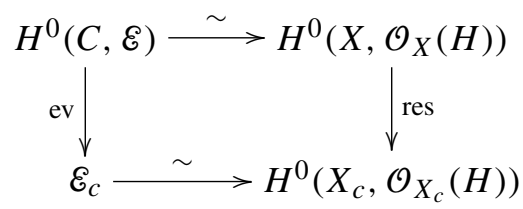

shows that $\mathscr{E}$ is spanned by global sections, since the restriction map res is surjective for any $c \in C$. Consider also the canonical induced diagram

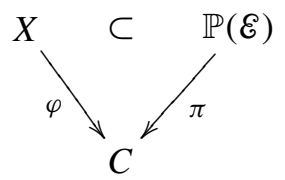

and write $X \sim 2 L+\pi^{*} B$, for some divisor $B$ on $C$. Let $H_{C}$ be a hyperplane section of $C \subset \mathbb{P}^{g-q-1}$. We find

$$
\varphi^{*}\left(H_{C}\right)=K+(r-1) H=\left.\left(K_{\mathbb{P}(\mathcal{E})}+X+(r-1) L\right)\right|_{X}=\varphi^{*}\left(K_{C}+\operatorname{det} \varepsilon+B\right) .
$$

By taking degrees, we get $g-1=2(q-1)+a+b$. Moreover, $a=\left(L^{r+1}\right)$, so $d=\left(L^{r} \cdot X\right)=2 a+b$. The two formulae follow. 
Lemma 7. Let $X \subset \mathbb{P}^{n}$ be smooth connected non-degenerate with $d \leqslant n$. Assume that the adjunction mapping $\varphi: X \rightarrow C$ makes $X$ into a quadric fibration over a smooth curve $C$. Then $C \simeq \mathbb{P}^{1}$.

Proof. Assume that $q=g(C)>0$. By Lemma 4 (ii), $d \geqslant 2 g+1$. So, by Lemma 6, we have $b=2(g-1)-d-4(q-1)<0$.

We show first that $\mathcal{E}$ is ample. As $\mathscr{E}$ is spanned, $\mathcal{O}_{\mathbb{P}(\mathscr{E})}(L)$ is spanned. So, if $L$ is not ample, there is a curve $D \subset \mathbb{P}(\mathscr{E})$ such that $(L \cdot D)=0$. It follows that $(X \cdot D)=\left(2 L+\pi^{*} B\right) \cdot D=\alpha b$ for some $\alpha>0$. As $b<0$, we deduce that $(X \cdot D)<0$, so $D \subset X$. But $\left.L\right|_{X}=H$, so $(D \cdot L)>0$ which is a contradiction. So $\mathcal{E}$ is ample.

Let now $S \subset X$ be a surface section of $X$. We have $\left(H_{S}+K_{S}\right)^{2}=0$, giving $d+2\left(H_{S} \cdot K_{S}\right)+\left(K_{S}\right)^{2}=0$. The adjunction formula yields $\left(H_{S} \cdot K_{S}\right)=2 g-2-d$. As $S$ is birationally ruled, it dominates a geometrically ruled model, say $S_{0}$. So, we have $\left(K_{S}\right)^{2} \leqslant\left(K_{S_{0}}\right)^{2}=8(1-q)$, see e.g. [12], Chapter V, Corollary 2.11 for the last equality. We deduce, using also Lemma 4 (ii)

$$
4(g-1) \geqslant d+8(q-1) \geqslant 2 g+1+8(q-1) .
$$

So we get $4 q \leqslant g+1$. By Lemma $6, a=1-g+2(q-1)+d$ and we find $a \leqslant d-2 q$. Now, since $\mathcal{E}$ is ample and spanned, we may apply Proposition 2 to find

$$
a=\operatorname{deg}(\mathscr{E}) \geqslant h^{0}(C, \mathcal{E})=h^{0}\left(X, \mathcal{O}_{X}(H)\right) \geqslant n+1 .
$$

Putting things together, we get

$$
n+1 \leqslant a \leqslant d-2 q \leqslant n-2 q .
$$

This is a contradiction, so $q=0$.

We shall also need the proposition below which might have an interest in itself.

Proposition 8. Let $X \subset \mathbb{P}^{n}$ be smooth connected non-degenerate and linearly normal. Assume that the adjunction mapping $\varphi: X \rightarrow C$ makes $X$ into a quadric fibration over $C \simeq \mathbb{P}^{1}$. Assume moreover, that $d \geqslant 2 g+2$ and $r \geqslant g+1$. Then, in the notation of Lemma 6 and denoting by $e=\left(e_{0}, \ldots, e_{r}\right)$ the splitting type of $\mathcal{E}$ and by $F$ a fibre of the projection $\mathbb{P}(\mathscr{E}) \rightarrow \mathbb{P}^{1}$, we have one of the following:

(a) $r=s, d=2 r, e=(1, \ldots, 1,0), X \in|2 L|$;

(b) $r=s-1, d=2 r+1, e=(1, \ldots, 1), X \in|2 L-F|$;

(c) $r=s-1, d=2 r, e=(1, \ldots, 1), X \in|2 L-2 F|$ or, equivalently, $X \simeq$ $\mathbb{P}^{1} \times Q^{r-1}$ embedded Segre;

(d) $r=s-2, d=2 r+2, e=(1, \ldots, 1,2), X \in|2 L-2 F|$; 
(e) $r=3, X \simeq \mathbb{P}^{1} \times \mathbb{F}_{1}$, embedded Segre, where $\mathbb{F}_{1}$ is embedded in $\mathbb{P}^{4}$ as a rational scroll of degree 3 .

Moreover, all these cases do occur.

Proof. We first remark that $g \geqslant 2$ (see [13]), so $r \geqslant 3$. Let $Q$ denote a fibre of $\varphi$. We have $(H-Q) \cdot H^{r-1}=d-2$. The standard exact sequence

$$
0 \longrightarrow \mathcal{O}_{X}(-Q) \longrightarrow \mathcal{O}_{X}(H-Q) \longrightarrow \mathcal{O}_{H}(H-Q) \longrightarrow 0
$$

and the fact that $H^{1}\left(X, \mathcal{O}_{X}(-Q)\right)=0$ allow one to prove by induction on $r$ that $|H-Q|$ is base-point-free. Note that on a curve section of $X$, the degree of the restriction of $|H-Q|$ is $\geqslant 2 g$, so it is base-point-free. Moreover, $|H-Q|$ is not composed with a pencil, since $r \geqslant 3$. So, by Bertini's theorem, there is a smooth member $X^{\prime} \in|H-Q|$. We let

$$
\begin{array}{ll}
H^{\prime}=\left.H\right|_{X^{\prime}}, & K^{\prime}=K_{X^{\prime}}, \\
r^{\prime}=\operatorname{dim}\left(X^{\prime}\right)=r-1, & \varphi^{\prime}=\varphi_{\left|K^{\prime}+\left(r^{\prime}-1\right) H^{\prime}\right|}, \\
d^{\prime}=\operatorname{deg}\left(X^{\prime}\right)=d-2, & g^{\prime}=g\left(H^{\prime}\right), \\
s^{\prime}=h^{0}\left(X^{\prime}, \mathcal{O}_{X^{\prime}}(H)\right)-1-r^{\prime} . &
\end{array}
$$

One finds easily $g^{\prime}=g-1, s^{\prime}=s-1$ and $\varphi^{\prime}$ can be identified with $\left.\varphi\right|_{X^{\prime}}$. The statement of the proposition is proved by induction on $r$ (note that we still have $d^{\prime} \geqslant 2 g^{\prime}+2$ and $\left.r^{\prime} \geqslant g^{\prime}+1\right)$. Assume first that $g \geqslant 3$. Since $r \geqslant g+1$, for $r=4$ we get $g=3$ and we may use the classification from Theorem 4.3 in [13]. For $r \geqslant 4$ we find inductively the following possible values for the numerical invariants:

(a) $r=s, d=2 r, g=r-1$;

(b) $r=s-1, d=2 r+1, g=r-1$;

(c) $r=s-1, d=2 r, g=r-2$;

(d) $r=s-2, d=2 r+2, g=r-1$.

It remains to analyze the case $g=2$, where one may use the classification theorem 3.4 in [13]. This leads to only one new case, which is (e).

Next we investigate the structure of $\mathscr{E}$ in each case.

First we have that $\mathscr{E}$ is non-special (since it is spanned by Lemma 6). So the Riemann-Roch theorem gives

$$
r+s+1=h^{0}(\mathcal{E})=a+r+1,
$$

hence $a=s$. Now, in case (a), we remark that $|H-2 Q|=\emptyset$, since $(H-Q)^{r-1}$. $(H-2 Q)=d-2 r-2<0$. By Lemma $6, b=0$, so $X \in|2 L|$. 
The exact sequence

$$
0 \longrightarrow \mathcal{O}_{\mathbb{P}(\mathcal{E})}(-L-2 F) \longrightarrow \mathcal{O}_{\mathbb{P}(\mathcal{E})}(L-2 F) \longrightarrow \mathcal{O}_{X}(H-2 Q) \longrightarrow 0
$$

shows that $h^{0}(\mathscr{E}(-2))=0$; as $\mathscr{E}$ is spanned and $a=r$, the splitting type of $\mathscr{E}$ must be $(1, \ldots, 1,0)$. The existence follows by the same type of argument as in the proof of Proposition 3 from [16]. The other cases are similar and simpler. For instance, in case (b) one gets as above $h^{0}(\mathscr{E}(-2))=0, a=r+1$ and $b=-1$. So $e=(1, \ldots, 1)$, $\mathcal{E}$ is very ample and the existence follows now easily.

Proposition 9. Let $X \subset \mathbb{P}^{n}$ be smooth connected non-degenerate and linearly normal, with $d \leqslant n$. Assume that the adjunction mapping makes $X$ into a quadric fibration over a smooth curve $C$. Then $X$ is as in case (ii) (b) or case (iv) of the main theorem.

Proof. By Lemma 7, $C \simeq \mathbb{P}^{1}$. We have $d \geqslant 2 g+1$ and $g \leqslant r-1$ by Lemma 4 . If $d \geqslant 2 g+2$, we may apply Proposition 8, thus leading to cases (ii) (b) and (iv) (b) up to (iv) (e) of the main theorem. So, assume that $d=2 g+1$. As in the proof of Proposition 8 we deduce that $a=s$. By Lemma 6 we get $a=g, b=1$. It follows $s=g \leqslant r-1$. Barth's theorem ([2]) ensures that $s \geqslant r-1$, so we must have $s=r-1$. We obtain

$$
g=r-1, \quad d=2 r-1, \quad a=r-1 .
$$

As in the proof of Proposition 8, we have $|H-2 Q|=\emptyset$, so $h^{0}(\mathscr{E}(-2))=0$. It follows that the splitting type of $\mathscr{E}$ is $(1, \ldots, 1,0,0)$, so we are in case (iv) (a) of the main theorem. The existence follows from Proposition 3 in [16].

We are now ready for the proof of our theorem.

Assume first that $r \leqslant s+1$. We have

$$
\Delta=d+r-h^{0}\left(X, \mathcal{O}_{X}(H)\right) \leqslant n+r-n-1=r-1 .
$$

If $\Delta=0$, by Theorem A we get either case (iii) of the main theorem or some special examples of case (i). Similarly if $\Delta=1$, by Theorem B we get either case (ii) (a) or some special examples of case (i). So, assume $\Delta \geqslant 2$, hence $r \geqslant 3$, from now on. If $r=3$, it follows $\Delta=2, s \geqslant 2$ and $\varphi: X \rightarrow \mathbb{P}^{1}$ is a quadric fibration by [13], Theorem 3.12 and Corollary 3.3. If $r=4$, we get $\Delta=2$ or $3, s \geqslant 3$, so $\varphi$ is either a quadric fibration over a rational curve or a scroll over $\mathbb{P}^{2}$ (see [13], Theorems 3.12, 4.8 and 4.2). Since $d \leqslant n$, it follows that $d \leqslant r+s \leqslant 2 s+1$. So, using the general properties of the adjunction mapping (see e.g. [4], Chapters 9-11, in particular Theorem 11.2.4) and the above analysis for $r \leqslant 4$, it follows from Theorem I in [15] that one of the following holds: 
(1) $X$ is a scroll over a (smooth) curve $C$;

(2) $\varphi$ makes $X$ into a scroll over a smooth surface;

(3) $\varphi$ makes $X$ into a quadric fibration over a smooth curve.

In case (1), from Corollary 3 , we get $C \simeq \mathbb{P}^{1}$, so $\Delta=0$. In case (2), by Proposition 5 we reach case (ii) (c). If we are in case (3), by Proposition 9 we get case (ii) (b) or case (iv). Assume now that $r \geqslant s+2$. By Barth's theorem ([2]) it follows that $\operatorname{Pic}(X) \simeq \mathbb{Z}$, generated by the class of $\mathcal{O}_{X}(H)$. We show that $X$ is Fano, so we are in case (i) and the main theorem is completely proved. As we have $\operatorname{Pic}(X) \simeq \mathbb{Z}$, to prove that $X$ is Fano it is enough to see that the geometric genus of $X$, denoted by $p_{g}$, is zero. Here we make use of a theorem of Harris (see [10]), generalizing Castelnuovo's bound for the genus of a curve to arbitrary dimension, which states that

$$
p_{g} \leqslant\left(\begin{array}{c}
M \\
r+1
\end{array}\right) s+\left(\begin{array}{c}
M \\
r
\end{array}\right) \varepsilon,
$$

where $M=[(d-1) / s]$ and $\varepsilon=d-1-M s$.

If $s=1$ we find $p_{g}=0$ by direct computation. If $s \geqslant 2$ and $r \geqslant 2$ we get $r+s-1<r s$; our hypothesis $d \leqslant r+s$ gives $d-1<r s$, or $M<r$. So $p_{g}=0$.

Proposition 10. Let $X \subset \mathbb{P}^{n}$ be a connected non-degenerate linearlynormal manifold with $d \leqslant n$. Assume moreover $s+2 \leqslant r \leqslant 2 s$ and $\Delta \geqslant 2$. Then one of the following holds:

(i) $r=4, d=6, X$ is a complete intersection of type (2, 3), or $5 \leqslant r \leqslant 6, d=8$, $X$ is a complete intersection of type $(2,2,2)$;

(ii) $r=6, d=10, X=C(G) \cap Q^{9} \subset \mathbb{P}^{10}$, where $G \subset \mathbb{P}^{9}$ is the Plücker embedding of the Grassmannian of lines in $\mathbb{P}^{4}, C(G)$ is the cone over $G$ and $Q^{9} \subset \mathbb{P}^{10}$ is a quadric;

(iii) $7 \leqslant r \leqslant 10, d=12, X$ is the spinorial variety $S^{10} \subset \mathbb{P}^{15}$, or one of its linear sections;

(iv) $r=8, d=14, X \subset \mathbb{P}^{14}$ is the Plücker embedding of the Grassmannian of lines in $\mathbb{P}^{5}$.

Proof. As above, $X$ is Fano and $\operatorname{Pic}(X)$ is generated by the class of $\mathcal{O}_{X}(H)$, so we may write $\mathcal{O}_{X}(-K) \cong \mathcal{O}_{X}(i H)$ for some $i>0$. The adjunction formula yields

$$
2 g-2=(r-i-1) d .
$$

We recall Castelnuovo's bound $g \leqslant M(d-((M+1) / 2) s-1)$ where $M=[(d-1) / s]$. Since $d \leqslant r+s \leqslant 3 s$, we find $M \leqslant 2$. Assuming $r-i-1 \geqslant 2$ we reach a 
contradiction; so $i \geqslant r-2$. But $\Delta \geqslant 2$ gives $g \geqslant 2$, so $i=r-2, X$ is a Mukai manifold and the result follows from [18].

Let us recall from [11] that the Hartshorne Conjecture predicts that when $r>2 s$, $X$ must be a complete intersection.

Corollary 11. Assume that the Hartshorne Conjecture holds for Fano manifolds. Then, in case (i) of the main theorem, $X$ is either a complete intersection or one of the varieties described in Theorem A, Theorem B, or Proposition 10.

Acknowledgements. I thank Francesco Russo for sending me the interesting paper [1] in preprint form and especially for explicitly asking me if I knew examples of (non-degenerate) irregular $r$-dimensional manifolds in $\mathbb{P}^{2 r+1}$, of degree $\leqslant 2 r+1$. In fact, his question was the starting point of the present work.

I thank the referee for his remarks that helped improving the presentation.

When writing the present version of the paper, I was supported by the Italian programme "Incentivazione alla mobilità di studiosi stranieri e italiani residenti all'estero".

\section{References}

[1] A. Alzati, F. Russo, Special subhomaloidal systems of quadrics and varieties with one apparent double point. Math. Proc. Cambridge Philos. Soc. 134 (2003), 65-82. Zbl 1057.14045 MR 1937793

[2] W. Barth, Transplanting cohomology classes in complex-projective space. Amer. J. Math. 92 (1970), 951-967. Zbl 0206.50001 MR 0287032

[3] W. Barth, Larsen's theorem on the homotopy groups of projective manifolds of small embedding codimension. In Algebraic geometry, Proc. Sympos. Pure Math. 29, Amer. Math. Soc., Providence, R.I., 1975, 307-313. Zbl 0309.14017 MR 0377123

[4] M. C. Beltrametti, A. J. Sommese, The Adjunction Theory of Complex Projective Varieties. De Gruyter Exp. Math. 16, de Gruyter, Berlin 1995. Zbl 0845.14003 MR 1318687

[5] G. Elencwajg, Les fibrés uniformes de rang 3 sur $\mathbb{P}^{2}(\mathbb{C})$ sont homogènes. Math. Ann. 231 (1978), 217-227. Zbl 0378.14003 MR 0481133

[6] T. Fujita, On the structure of polarized manifolds with total deficiency one, I. J. Math. Soc. Japan 32 (1980), 709-725. Zbl 0474.14017 MR 0589109

[7] T. Fujita, On the structure of polarized manifolds with total deficiency one, II. J. Math. Soc. Japan 33 (1981), 415-434. Zbl 0474.14018 MR 0620281

[8] W. Fulton, R. Lazarsfeld, Connectivity and its applications in algebraic geometry. In Algebraic geometry (Chicago, Ill., 1980), Lecture Notes in Math. 862, Springer-Verlag, Berlin 1981, 26-92. Zbl 0484.14005 MR 0644817

[9] T. Gaffney, R. Lazarsfeld, On the ramification of branched coverings of $\mathbb{P}^{n}$. Invent. Math. 59 (1980), 53-58. Zbl 0422.14010 MR 0575080 
[10] J. Harris, A bound on the geometric genus of projective varieties. Ann. Scuola Norm. Sup. Pisa 8 (1981), 35-68. Zbl 0467.14005 MR 0616900

[11] R. Hartshorne, Varieties of small codimension in projective space. Bull. Amer. Math. Soc. 80 (1974), 1017-1032. Zbl 0304.14005 MR 0384816

[12] R. Hartshorne, Algebraic Geometry. Springer-Verlag, 1977. Zbl 0367.14001 MR 0463157

[13] P. Ionescu, Embedded projective varieties of small invariants. I. In Proceedings of the Week of Algebraic Geometry, Bucharest, 1982, Lecture Notes in Math. 1056, Springer-Verlag, Berlin 1984, 142-186. Zbl 0542.14024 MR 0749942

[14] P. Ionescu, Embedded projective varieties of small invariants. III. In Algebraic Geometry (L'Aquila 1988), Lecture Notes in Math. 1417, Springer-Verlag, Berlin 1990, 138-154. Zbl 0709.14023 MR 1040557

[15] P. Ionescu, On varieties whose degree is small with respect to codimension. Math. Ann. 271 (1985), 339-348. Zbl 0541.14032 MR 0787185

[16] P. Ionescu, M. Toma, Boundedness for some special families of embedded manifolds. In Classification of Algebraic Varieties (L'Aquila 1992), Contemp. Math. 162, Amer. Math. Soc., Providence, R.I., 1994, 215-225. Zbl 0848.14001 MR 1272700

[17] J. Kollár, Y. Miyaoka, S. Mori, Rationally connected varieties. J. Alg. Geom. 1 (1992), 429-448. Zbl 0780.14026 MR 1158625

[18] S. Mukai, Biregular classification of Fano 3-folds and Fano manifolds of coindex 3. Proc. Nat. Acad. Sci. USA 86 (9) (1989), 3000-3002. Zbl 0679.14020 MR 0995400

Received February 1, 2007

Paltin Ionescu, University of Bucharest, Faculty of Mathematics and Computer Science, 14 Academiei Str., 010014 Bucharest

and

Institute of Mathematics of the Romanian Academy, P.O. Box 1-764, 014700 Bucharest,

Romania

E-mail: Paltin.Ionescu@imar.ro 